\title{
Web of Well-Being: Re-Examining PERMA and Subjective Well-Being Through Networks
}

\author{
Sean H. Merritt ${ }^{1}$, Saeideh Heshmati ${ }^{1}$, Zita Oravecz ${ }^{2}$ and Stewart I. Donaldson ${ }^{1}$ \\ School of Social Science, Policy, and Evaluation, Claremont Graduate University ${ }^{1}$ \\ Human Development and Family Studies, Pennsylvania State University ${ }^{2}$ \\ This Pre-print is under review at the Journal of Positive Psychology
}

\section{Data Availability Statement}

The data that support the findings of this study are openly available in osf at http://doi.org/10.17605/osf.io/h2d4s, reference number 10.17605/osf.io/h2d4s.

\section{Author Note}

Correspondence should be sent to Sean Merritt, Claremont Graduate University, 150 E 10th St, Claremont, CA91711 E-mail: sean.merritt@cgu.edu 


\begin{abstract}
While positive psychology has been in development for more than 20 years, positive psychologists still face the challenge of finding optimal measurements of well-being. The PERMA framework provides a new understanding of well-being based on years of research. However, whether it adds value in terms of being distinct from the already established measures of Subjective Well-being (SWB) has recently been under debate. Certain characteristics of PERMA make it unsuited for traditional factor analysis and data analysis techniques. In this paper, we argue that a network approach is more suitable for understanding the interrelated nature of PERMA components. We then show how the components of PERMA and SWB are distinct using exploratory graphical analysis. Our results show that while PERMA predicts SWB, PERMA provides us a more nuanced way to understand the interrelated antecedents of well-being that can be developed, which we refer to as the web of well-being.

Key-words: PERMA; Subjective Well-being; Well-being; Psychometrics; Network Analysis
\end{abstract}




\section{Web of Well-Being: Re-Examining PERMA and Subjective Well-Being Through Networks}

The advent of Positive Psychology (Seligman \& Csikszentmihalyi, 2000) brought a pronounced focus on the scientific investigation of subjective well-being (Diener et al., 1999). Consequently, various theoretical approaches to understanding and conceptualizing well-being have been proposed by positive psychologists. One recent multidimensional framework was proposed by Seligman (2011), who argued that well-being is composed of five building blocks: Positive emotions, Engagement, Relationships, Meaning, and Accomplishment (PERMA). Since this proposition, PERMA has been the center of numerous research studies (e.g., Butler \& Kern, 2016; Donaldson et al., 2020; Heshmati et al. 2020, Slavin et al., 2012 ) and recently has been a central topic of debate: Goodman and colleagues (2018) found PERMA was highly correlated (latent correlation $=.98)$ with Subjective Well-Being (SWB; Deiner et al, 1999) and thus concluded that it was redundant. Seligman (2018) responded by noting that PERMA was not intended to measure a different type of well-being but rather to be complementary to SWB. Rather than PERMA measuring well-being directly, it is a measure of constructs that lead to well-being. He suggested that it can be used to disentangle the elements of well-being to be used selectively in intervention settings.

In his response, Selgiman (2018) proposes six criteria to evaluate the elements of well-being: 1) they contribute to well-being, 2) a person will pursue each element for its own sake, 3) the list is exclusive and exhausted, 4 ) the elements lead to specific interventions to build each element and SWB, 5) the list is parsimonious, and 6) each element can be defined and measured independently. Seligman (2018) points out that Goodman and colleagues (2018) results do cause some concern for the last requirement. He goes on to suggest three possible reasons. 
First is the conclusion that Goodman et al. (2018) came to, which is that each element is the same as SWB and therefore redundant. Second, is the possibility of a halo effect. That due to the constraint of self-reports, those feeling more positively rating other elements highly. The final explanation he offers is that there exist causal connections and third variable connections between these elements.

This paper will examine if PERMA meets requirement six using network psychometrics. Network psychometrics is similar to factor analysis in that it helps to understand the relationship among latent variables. However, network psychometrics makes different assumptions about the data leading to a different model structure and interpretation of results. With this approach, we (1) allow the elements of PERMA to form an interrelated "web of well-being" rather than independent "building blocks," and (2) can study the relationship between elements of PERMA and SWB with more specificity, considering them as networks of well-being. The results will help us address two important questions: (1) whether PERMA elements are distinct but interconnected in nature, testing the "exclusivity" and "connectivity" properties of PERMA as theorized by Seligman (2011, p. 16; 2018), and (2) whether considering the relationship between PERMA and SWB is due to redundancy of constructs or that PERMA are the elements of well-being.

\section{Advantages of Network Psychometrics}

Network psychometric analysis is a novel statistical tool that goes beyond traditional factor analysis methods. Network psychometric analysis represents constructs using network graphs, similar to those in social network analysis. As demonstrated in Figure 1, each item in a network is represented by a "node" and the relationships among items by "edges" or "links". 
The color and size of the edge relates to the size and direction of the relationship. Positive relationships are green and negative are red. Thicker edges mean a stronger relationship between two nodes. Representing constructs this way helps to identify important features of a network and how these features group together. For a more technical introduction see Marsman et al. (2017), Eskamp \& Fried (2018), and Kan et al. (2020).

\section{Figure 1.}

\section{Example of a three-node three-edge network}

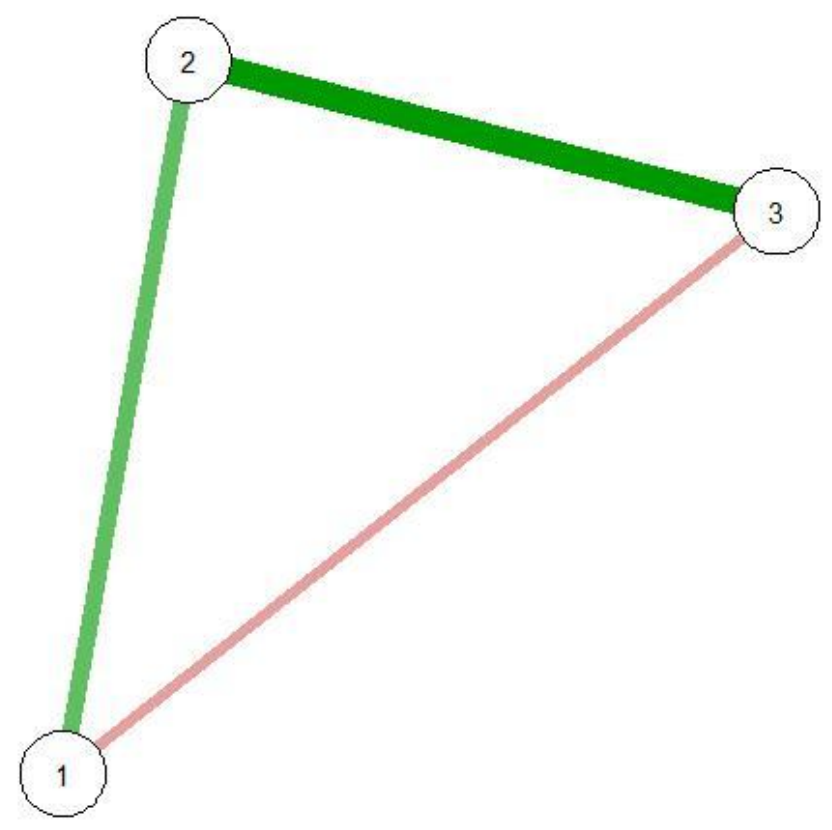

One advantage of network psychometrics is that the assumption of local independence is not required, as opposed to factor models. This approach has been helpful in understanding comorbidity of mental health (Borsboom \& Cramer, 2013; McNally et al., 2015), personality (Christensen et al., 2020), and intelligence (Schmank et al., 2019). These constructs highly 
correlate among sub-constructs and items, making results from factor analyses questionable.

However, with network analysis, we can examine the interrelations within a construct to better understand how these constructs emerge. Due to this, network models are better designed to extract the unique associations between variables (Christensen \& Golino, 2021; Cramer et al, 2012). Where latent correlations model all the common covariations, network models are able to reduce the variance shared due to the halo effect. Therefore, the majority of the halo effect in the data will be accounted for in the network models. Given the concern for a halo effect, a network approach may be more appropriate than factor analysis methods to disentangle the web of well-being formed by PERMA elements.

Network psychometrics also allow us to perform exploratory graphical analysis (EGA) and examine network loadings (Epskamp \& Golino, 2018; Christenson \& Golino, 2020). EGA is similar to exploratory factor analysis (EFA), in that it searches for the number of constructs by grouping items. However, EGA uses community detection algorithms, calculates network loadings, and does not require a pre-specified number of factors to split the common covariance (Christensen \& Golino, 2021). The biggest advantage EGA has over EFA is that it is able to detect unique factors when the correlation among factors is high. Based on the intercorrelated nature of elements of well-being demonstrated in previous research (Heshmati et al., 2020) EGA would be more appropriate. We start by determining the communities (statistically consistent with factors; Golino \& Epskamp, 2017) within the network using the Walktrap algorithm (Pons \& Latapy, 2005). Then we calculate network loadings with each corresponding community. A community can be considered a single measurable element. Therefore, any of the PERMA elements in the same cluster can be considered redundant. Network loadings are similar to factor 
loadings except they allow for meaningful cross-loadings with multiple factors.These cross-loadings can be interpreted as the contribution an item has to the development of a related variable. The primary assumption of these interpretations is that the construct is a network model. For a more thorough introduction to network loadings please see Christensen \& Golino (2020). If PERMA is truly no different from SWB then we would expect PERMA constructs to have high loadings on SWB and no more than three communities (SWL, positive affect, and negative affect). But if they are distinct we see seven communities (SWL, positive affect, negative affect, engagement, meaning, relationships, and accomplishment ). Similarly, if PERMA has distinct elements then we would expect five communities if modeled by itself (one for each element of well-being). If there are any causal relations between elements, like Seligman suggested, then we would expect to find cross-loadings between clusters.

To summarize, a network psychometric analysis approach is thus a useful approach to examine the probability of a "web" of well-being--in place of building blocks of well-being-with elements of PERMA as a woven web of elements that are intercorrelated. Using this technique we can examine the high correlations of PERMA. Networks modeling unique variance address reason 2 (halo effect). The network psychometric technique can then be used to examine network loadings among the PERMA items to determine the degree to which items belong to communities or groupings of items. This addresses reasons 1 (redundant measures) and 3 (causal and third variable connections).Through a network approach, PERMA elements may be proven to be conceptualized more as a web of elements of well-being that are interlinked rather than building blocks of well-being that build on top of each other, implying some features to be more foundational to the structure of well-being compared to others. 


\section{The Current Study}

Using a network perspective, in the current study, we examined SWB and PERMA in a different light. Seligman (2011) called PERMA the "building blocks" of wellbeing--an idea that might stem from the limitation imposed by traditional latent factor models. Thinking of PERMA with factor models creates the image of physical blocks -- you stack those blocks up and it makes up well-being. Looking at PERMA with a network paradigm changes the idea of building blocks to a web of interconnected entities (or dependent constructs). Through this perspective, we theorize well-being via constructs that work together to determine a person's well-being as a network. We may also take this further and say that well-being is a summary of these behaviors

(PERMA) that tend to co-occur and associate with well-being (Cramer, 2012). In this study, we show how PERMA can be thought of as a network. We also show how this paradigm integrates PERMA into subjective well-being theory. For this purpose, in the current study, we aim to answer the following research questions:

1. Does the conceptualization of PERMA elements as a web of well-being offer new information on its structure?

2. Are the features of PERMA distinct from those of SWB (SWL, positive and negative emotions)?

3a. Are the elements of PERMA distinct from each other?

3b. How are the PERMA elements related to each other?

In this paper, we examine the overlap of SWB and PERMA measures. First, we model PERMA as a network and check indices for proper fit. Similar to factor analysis, networks provide fit indices to determine how well the hypothesized models match the data. We 
hypothesize PERMA will fit a network model as shown in previous research in an early adult population (Heshmati et al., 2020). Here, we also fit PERMA networks with two additional constructs (health and negative emotions) included in the PERMA Profiler measure (Butler \& Kern, 2016). (H1) We hypothesize these constructs will indicate a good fit when examining PERMA as a network. Finally, we examine the clusters and network loadings of a combined network of PERMA and SWB. With this we are able to test if the components of PERMA are distinct from SWB, distinct from other PERMA elements, and further examine how these elements are related to each other. H2: We hypothesize that PERMA will cluster separately from SWL and PANAS and will have little to no cross-loadings, indicating that PERMA measures components of well-being that are distinct from the SWB. We further hypothesize that (H3a) PERMA will cluster separately, but (H3b) will show cross-loadings between clusters, suggesting potential causal relationships between elements.

\section{Method}

\section{Participants and Procedure}

Using Prolific's stratified sampling via 'prolific.co', we obtained a representative sample of 580 US adults, according to age $(M=46.49, \mathrm{SD}=16.04)$, gender $($ Male $=272$, Female $=278$, other $=11$, and ethnicity $($ white $=435$, black $=72$, Hispanic $=35$, Asian $=35$, Native American $=8$, Pacific Islander $=2$, other $=4$ ) matching the U.S. census. Prolific is a specialized survey hosting platform that verifies and monitors participants with sophisticated checks for high-quality data. Their services include representative sampling based on the demographics listed previously. A call for participants was listed on the site where those who were interested were redirected to our survey hosted on Qualtrics. After receiving consent, participants filled out 
the PERMA Profiler, Satisfaction with Life Scale, PANAS, BFI-2S, and demographics questions. After completing the survey, participants were paid for their time. While Prolific had pre-recorded individuals' age, gender, and ethnicity, we gathered the same demographic info to verify representativeness. We also asked about education, occupation, relationship status, religion, and the number of family members living in the home. This process was approved by the Claremont Graduate University Institutional Review Board (Protocol ID: 3952)

\section{Measures}

\section{Subjective Well-Being}

To measure subjective well-being, we used Diener's (Kobau et al., 2010) 5-item Satisfaction with Life Scale (SWLS; alpha =.93) and the 13-item Positive Affect and Negative Affect Schedule (PANAS; pos: alpha $=.90$, neg: alpha $=.87$ ). SWLS asks participants to make cognitive assessments about their satisfaction with their life (i.e. in most ways my life is close to my ideal) using a 7-point Likert scale ( $1=$ strongly disagree to $7=$ strongly agree). The PANAS asks participants to rate how frequently they have experienced positive and negative emotions (e.g. joyful, afraid, and pleasant) using a 5-point Likert scale ( $1=$ very rarely or never to $5=$ very often or always). The scale is also designed to change the relative time period for participants to consider their emotions. For the purpose of our study, we asked them to rate the last four weeks.

\section{PERMA Profiler}

We used the PERMA Profiler (Butler \& Kern, 2016) to measure PERMA. This scale contains three items for Positive emotions (e.g., In general, how often do you feel joyful?), Engagement (e.g., How often do you become absorbed in what you are doing?), Relationships (e.g., To what extent do you receive help and support from others when you need it?), Meaning 
(e.g., In general, to what extent do you lead a purposeful and meaningful life?), and Accomplishment (e.g., How much of the time do you feel you are making progress towards accomplishing your goals?). Cronbach's Alpha for the PERMA Profiler, measuring the five elements of PERMA was alpha $=.90$. In addition to the five PERMA elements, PERMA Profiler also contains three questions on negative affect (e.g., In general, how often do you feel anxious?), three questions on health (e.g., In general, how would you say your health is? ), a loneliness question (How lonely do you feel in your daily life?), and a happiness question (Taking all things together, how happy would you say you are?). While we asked all 23 questions we used the first 21 questions, excluding the items on happiness and loneliness, for our analysis of PERMA. We did this because these questions were single-item measures, and might not provide valid measures of the two constructs.

\section{Data Analysis}

To address hypothesis one, we tested the fit of items measuring PERMA in a network model through psychometric network analysis. Specifically, using the psychonetrics package (version 0.10; Kan et al., 2020) in the R statistical software (R Core Team, 2018), we modeled the data from the PERMA questionnaire. R code can be found at osf.io/h2d4s/. Specifically, we split our data in half, the first half was used for exploratory analysis and the other half for confirmatory to avoid overfitting (Fokkema \& Grieff, 2017). Following the tutorial as outlined in Kan et al. (2020), we started by calculating the partial correlation matrix (Borsboom \& Cramer, 2013; Epskamp \& Fried, 2016; McNally et al., 2015) on the exploratory data. To prevent spurious correlations, we removed those with a p-value greater than .01 . We then extracted the adjacency matrix and used it to test the fit of the confirmatory data. Confirmatory network 
psychometrics use the same fit criteria as confirmatory factor analysis (see Schermelleh-Engel et al., 2003).

To examine hypotheses 2 through $3 \mathrm{~b}$ we examined overlapping clusters and their corresponding network loadings within the measures of PERMA and SWB using exploratory graphical analysis (EGA; Golino \& Epskamp, 2017; Golino et al., 2020) with the EGAnet package (version 1.2.0). Network loadings for a node are calculated by taking the sum of the edges connected to it within each cluster and then standardized by dividing by the square root of the sum of loadings for a given cluster. For more details see Christensen and Golino (2020).

\section{Results}

\section{Descriptive Analysis}

Using Pearson correlations we found that all of the constructs were highly correlated (see table 1); the only exception being engagement. Where most correlations ranged between .50 and .87 , engagement correlated with other constructs in the .20 to .42 range.

\section{Table 1}

Descriptives and Correlations

\begin{tabular}{|c|c|c|c|c|c|c|c|c|c|c|c|c|}
\hline & $\mathrm{M}$ & SD & 1 & 2 & 3 & 4 & 5 & 6 & 7 & 8 & 9 & 10 \\
\hline 1. P & 5.85 & 2.41 & - & & & & & & & & & \\
\hline 2. E & 6.80 & 1.91 & $\begin{array}{c}.45 * * \\
*\end{array}$ & - & & & & & & & & \\
\hline 3. $\mathrm{R}$ & 6.38 & 2.51 & $\begin{array}{c}.72 * * \\
*\end{array}$ & $\begin{array}{c}.33 * * \\
*\end{array}$ & - & & & & & & & \\
\hline 4. M & 6.18 & 2.64 & $\begin{array}{c}.78^{* *} \\
*\end{array}$ & $\begin{array}{c}.42 * * \\
*\end{array}$ & $.63 * * *$ & - & & & & & & \\
\hline 5. A & 6.83 & 2.05 & $\begin{array}{c}.72 * * \\
*\end{array}$ & $\begin{array}{c}.37 * * \\
*\end{array}$ & $.62 * * *$ & $\begin{array}{c}.79 * * \\
*\end{array}$ & - & & & & & \\
\hline 6. $\mathrm{N}$ & 4.22 & 2.46 & $\begin{array}{c}-.59^{*} \\
* *\end{array}$ & $\begin{array}{c}-.20^{*} \\
* *\end{array}$ & $\begin{array}{c}-.48^{* *} \\
*\end{array}$ & $\begin{array}{c}-.54^{*} \\
* *\end{array}$ & $\begin{array}{c}-.57^{*} \\
* *\end{array}$ & - & & & & \\
\hline
\end{tabular}




\begin{tabular}{|c|c|c|c|c|c|c|c|c|c|c|c|c|}
\hline 7. $\mathrm{H}$ & 6.32 & 2.32 & $\begin{array}{c}.59 * * \\
*\end{array}$ & $\begin{array}{c}.28 * * \\
*\end{array}$ & $.48 * * *$ & $\begin{array}{c}.54 * * \\
*\end{array}$ & $\begin{array}{c}.58^{* * *} \\
*\end{array}$ & $\begin{array}{c}-.41^{*} \\
* *\end{array}$ & - & & & \\
\hline $\begin{array}{l}\text { 8.SW } \\
\mathrm{L}\end{array}$ & 3.63 & 1.73 & $\begin{array}{c}.69^{* *} \\
*\end{array}$ & $\begin{array}{c}.25^{* *} \\
*\end{array}$ & $.62 * * *$ & $\begin{array}{c}.64^{* *} \\
*\end{array}$ & $\begin{array}{c}.62 * * \\
*\end{array}$ & $\begin{array}{c}-.46^{*} \\
* *\end{array}$ & $\begin{array}{c}.49 * * \\
*\end{array}$ & - & & \\
\hline 9.Pos & 23.62 & 5.63 & $\begin{array}{c}.87 * * \\
*\end{array}$ & $\begin{array}{c}.41 * * \\
*\end{array}$ & $.64 * * *$ & $\begin{array}{c}.73 * * \\
*\end{array}$ & $\begin{array}{c}.69 * * \\
*\end{array}$ & $\begin{array}{c}-.63^{*} \\
* *\end{array}$ & $\begin{array}{c}.53^{* *} \\
*\end{array}$ & $\begin{array}{c}.61^{* *} \\
*\end{array}$ & - & \\
\hline $\begin{array}{l}10 . \\
\text { Neg }\end{array}$ & 13.81 & 4.90 & $\begin{array}{c}-.65^{*} \\
* *\end{array}$ & $\begin{array}{c}-.28^{*} \\
* *\end{array}$ & $\begin{array}{c}-0.52^{*} \\
* *\end{array}$ & $\begin{array}{c}-.60^{*} \\
* *\end{array}$ & $\begin{array}{c}-.61^{*} \\
* *\end{array}$ & $\begin{array}{c}.77 * * \\
*\end{array}$ & $\begin{array}{c}-.47^{*} \\
* *\end{array}$ & $\begin{array}{c}-.50^{*} \\
* *\end{array}$ & $\begin{array}{c}-.67^{*} \\
* *\end{array}$ & - \\
\hline
\end{tabular}

\section{Network Fitting}

In the next round, we conducted psychometric network analysis on measures of PERMA and SWB. We first constructed the PERMA network (Figure 2) using items from the PERMA Profiler that measure each of the five PERMA elements. This analysis tested our hypothesis that well-being could be explained as a network. Figure 2 shows how the PERMA network items within the same constructs (Positive emotions, Engagement, Relationship, Meaning, and Accomplishment) tend to have stronger relations than items between constructs. 
Figure 2.

PERMA Network

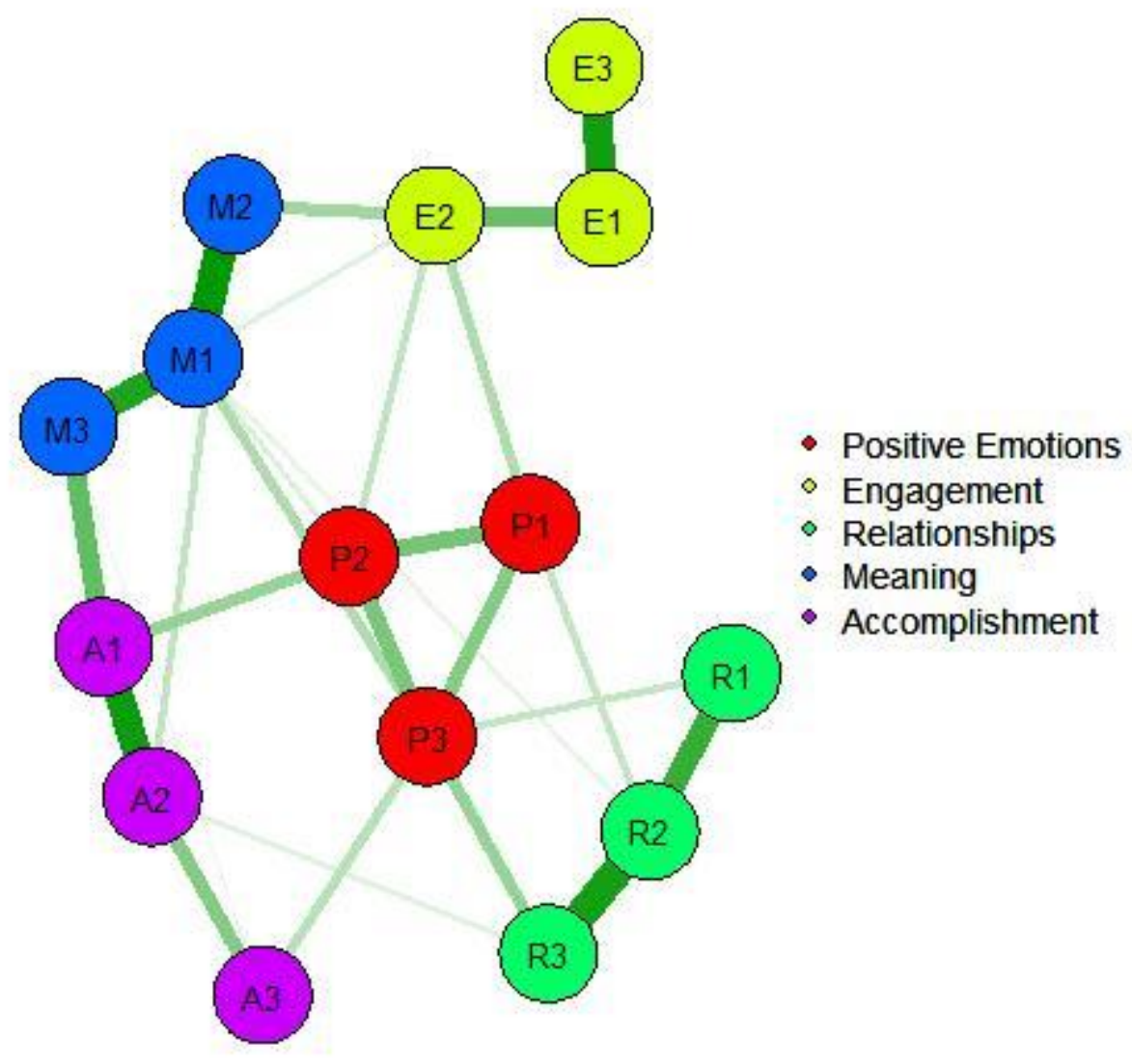

Note: Each node represents an item of PERMA. Colors are used to help distinguish each factor of PERMA. Green edges represent a positive relationship and thicker edges are stronger relationships. 
We then added negative emotions and health to the initial PERMA network

$(\mathrm{PERMA}+\mathrm{NH}$; Figure 3$)$ for insight into the impact of additional factors on the fit and clustering of the network. Looking at the network we see stronger relationships within each component.

Figure 3.

PERMA+NH Network

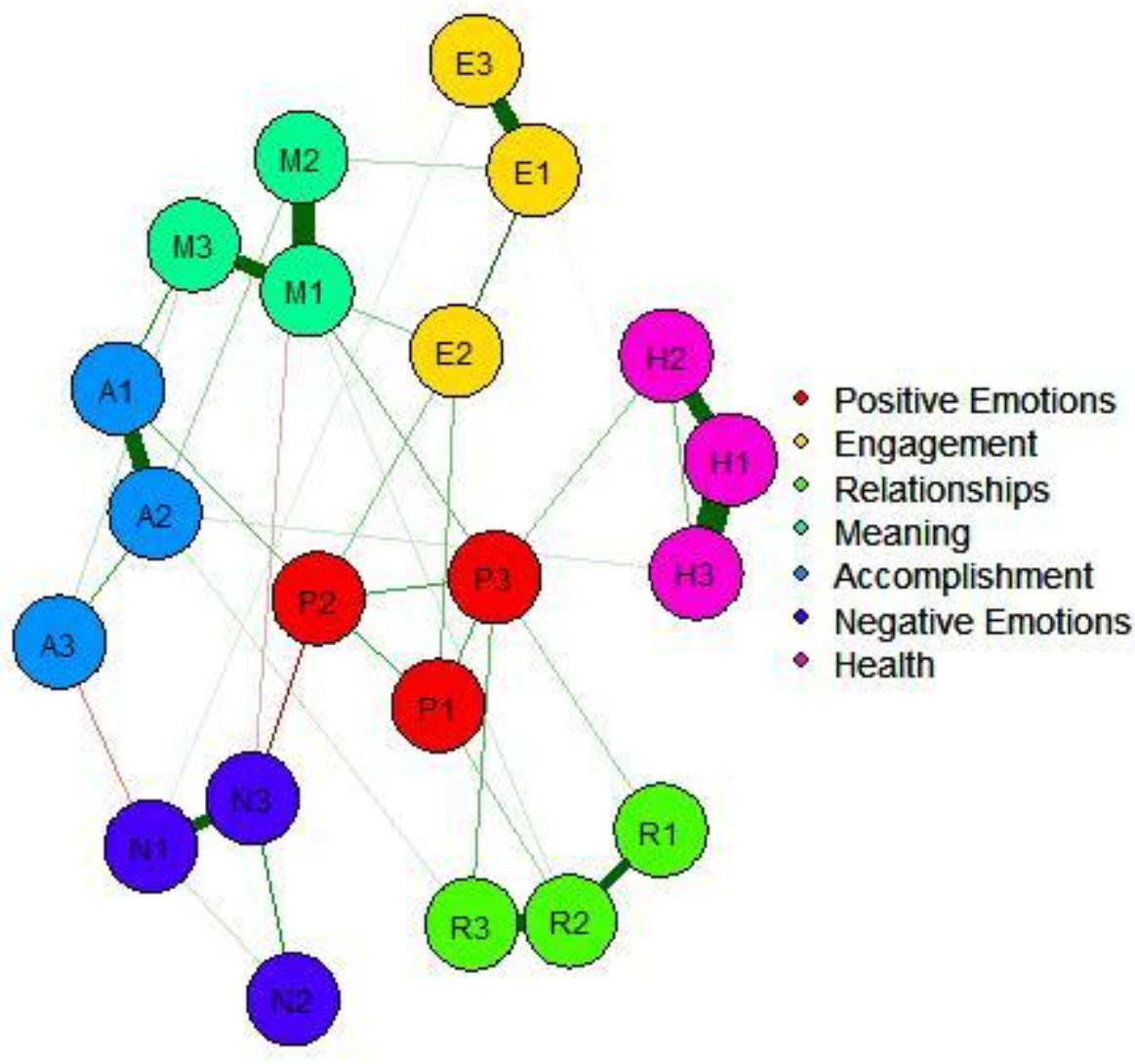

Note: Each node represents an item of PERMA. Colors are used to help distinguish each factor of PERMA. Green edges represent a positive relationship and thicker edges are stronger relationships. 
Finally, we combined PERMA+NH and SWB to understand the relationship between the elements SWB and PERMA (Figure 4). Figure 4 is a much more complex network, but we can still observe that strong relationships exist within constructs with a variety of edges connecting them. To better understand the network in figure 4 we used Exploratory Graphical Analysis (EGA) to dissect the relationships among items. The results of the EGA are elaborated on in the following section.

\section{Figure 4.}

\section{PERMA $+N H+S W B$ Network}

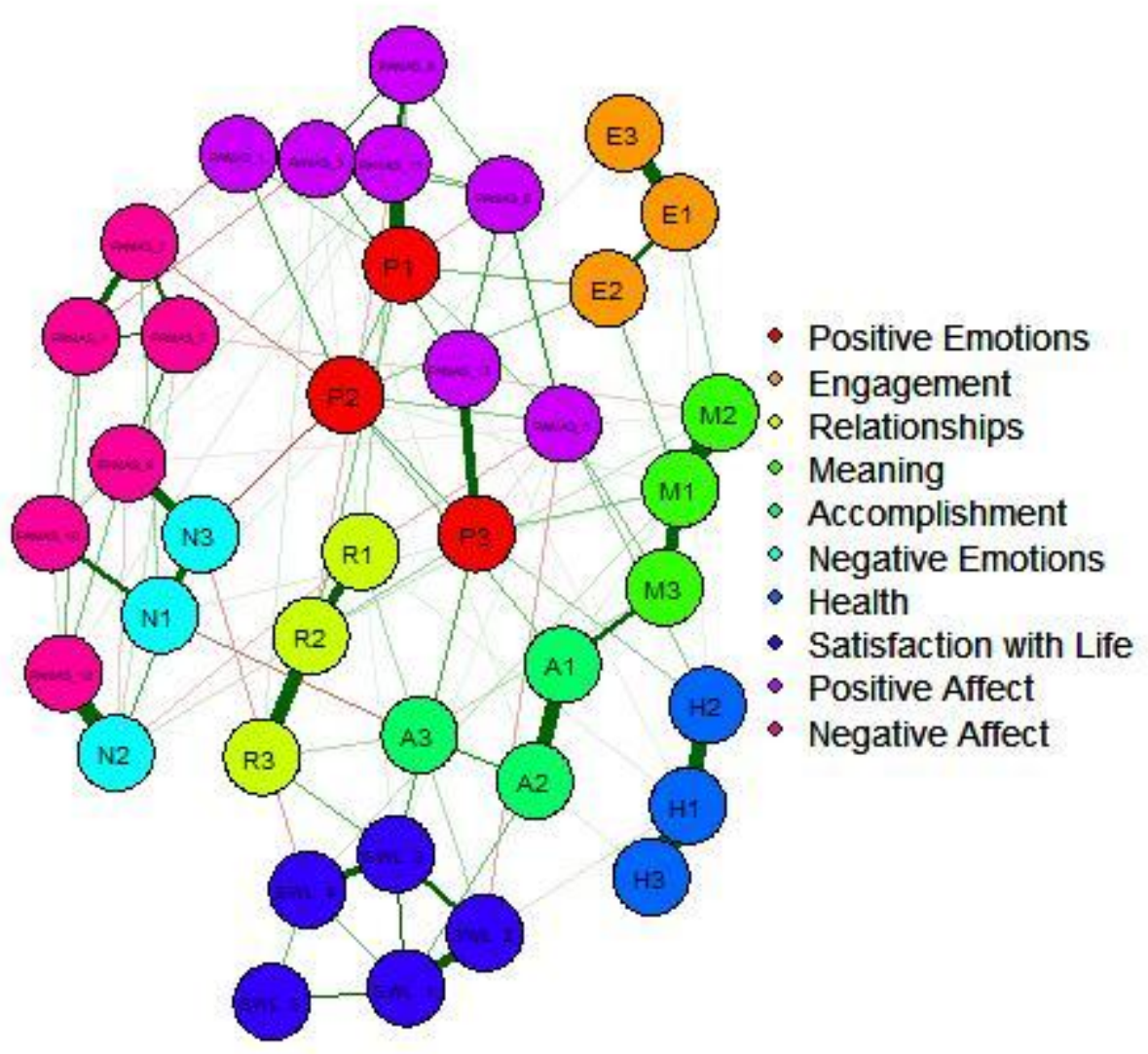


Note: Each node represents an item of PERMA. Colors are used to help distinguish each factor of PERMA. Green edges represent a positive relationship and thicker edges are stronger relationships.

After these models were constructed, we examined the fit indices. These are the same fit indices used for CFA models. All models had an excellent fit (see Table 2), showing that not only PERMA including only the five core elements can be represented as a network, but even when Negative emotions and Health $(\mathrm{PERMA}+\mathrm{NH})$ as well as SWB $(\mathrm{PERMA}+\mathrm{NH}+\mathrm{SWB})$ is added to the model, all models are robust and can be represented as networks.

\section{Table 2}

Fit Indices for Networks

\begin{tabular}{lcccccccc}
\hline Model & $\mathrm{df}$ & $\chi^{2}$ & TLI & CFI & RMSEA & AIC & BIC & EBIC \\
& & & & & & & & \\
\hline PERMA & 176 & 147.44 & 0.97 & 0.98 & 0.06 & 8474.73 & 8634.82 & 9111.43 \\
PERMA+NH & 170 & 364.56 & 0.95 & 0.96 & 0.06 & 11736.58 & 11958.52 & 12701.38 \\
PERMA+NH+SWB & 633 & 1134.25 & 0.95 & 0.95 & 0.05 & 20825.65 & 21360.48 & 23514.66 \\
\hline
\end{tabular}

\section{Network Loadings and Clustering}

Finally, we used EGA to test how items found in PERMA and SWB clustered together. If PERMA is redundant to SWB then we will expect to see PERMA and SWB to cluster or have high network loadings on each other. For this part of the analysis, we only performed EGA on the PERMA+NH+SWB model as we were primarily interested in whether or not PERMA and SWB clustered together. EGA provides network loadings for how much an item is connected to a given cluster. Table 3 shows the network loadings for each item. Network loadings are similar to 
CFA loadings with the exception that items can be loaded onto multiple groups, indicating interrelations among groups (Christensen \& Golino, 2020). We found seven distinct clusters within the network. We will label these negative emotions (1), meaning \& achievement (2), engagement (3), positive emotions (4), relationships (5), SWL (6), and health(7) respectively. Meaning and achievement were the only factors among PERMA+NH to load onto a combined cluster, suggesting that meaning and achievement are not independent. SWL, health, relationships, and engagement clustered within their respective groups. Negative emotions (PERMA) clustered with negative affect (PANAS). Positive emotions tended to be equally clustered with positive affect and meaning and accomplishment. These clustering groups show that PERMANH factors are distinct from the factors of SWB (with the exception of positive and negative emotions). If they were not distinct, we would expect more of PERMANH to cluster with positive or negative affect and satisfaction with life. Further interpretations of these loadings depends on the assumptions that it is a network model. Given the results from our fit indices we find this assumption likely. Furthermore, bootstrapping the EGA we find these results to be stable (standard error $=0.000)$.

Table 3

Network Loadings

\begin{tabular}{|c|c|c|c|c|c|c|c|}
\hline & 1 & 2 & 3 & 4 & 5 & 6 & 7 \\
\hline P1 & - & .10 & - & .18 & - & - & - \\
\hline P2 & - & .13 & - & .13 & - & - & - \\
\hline P3 & - & .12 & - & .15 & - & - & - \\
\hline E1 & - & .14 & .34 & - & - & - & - \\
\hline E2 & - & .15 & .23 & .- & - & - & - \\
\hline E3 & - & - & .34 & - & - & - & - \\
\hline $\mathrm{R} 1$ & - & - & - & - & - & .29 & - \\
\hline $\mathrm{R} 2$ & - & - & - & - & - & .44 & - \\
\hline
\end{tabular}




\begin{tabular}{|c|c|c|c|c|c|c|c|}
\hline R3 & - & - & - & - & - & .28 & - \\
\hline M1 & - & .32 & - & - & - & - & - \\
\hline M2 & - & .25 & - & - & - & - & - \\
\hline M3 & - & .30 & - & - & - & - & - \\
\hline A1 & - & .27 & - & - & - & - & - \\
\hline A2 & - & .27 & - & - & - & - & - \\
\hline A3 & - & .11 & - & - & - & - & - \\
\hline N1 & .24 & - & - & - & - & - & - \\
\hline N2 & .24 & - & - & - & - & - & - \\
\hline N3 & .27 & - & - & - & - & - & - \\
\hline $\mathrm{H} 1$ & - & - & - & - & - & - & .53 \\
\hline $\mathrm{H} 2$ & - & - & - & - & - & - & .40 \\
\hline $\mathrm{H} 3$ & - & - & - & - & - & - & .48 \\
\hline SWL_1 & - & - & - & - & .46 & - & - \\
\hline SWL_2 & - & - & - & - & .32 & - & - \\
\hline SWL_3 & - & - & - & - & .41 & - & - \\
\hline SWL_4 & - & - & - & - & .29 & - & - \\
\hline SWL_5 & - & - & - & - & .21 & - & - \\
\hline PANAS_1 & - & - & - & .22 & - & - & - \\
\hline PANAS_2 & .29 & - & - & - & - & - & - \\
\hline PANAS_3 & - & - & - & .35 & - & - & - \\
\hline PANAS_4 & .33 & - & - & - & - & - & - \\
\hline PANAS_5 & - & - & - & .14 & - & - & - \\
\hline PANAS_6 & - & - & - & .28 & - & - & - \\
\hline PANAS_7 & .31 & - & - & - & - & - & - \\
\hline PANAS_8 & - & - & - & .38 & - & - & - \\
\hline PANAS_9 & .31 & - & - & - & - & - & - \\
\hline PANAS_10 & .21 & - & - & - & - & - & - \\
\hline PANAS_11 & - & - & - & .33 & - & - & - \\
\hline PANAS_12 & .32 & - & - & - & - & - & - \\
\hline PANAS_13 & - & .11 & - & .15 & - & - & - \\
\hline
\end{tabular}

\section{Discussion}

Here we used a network approach to better understand the underlying relationships between the PERMA constructs and SWB. Whereas factor analytic methods are useful for scale validation purposes, its assumption of independence is deemed to be inappropriate for the assessment of well-being measures that have been shown to be made up of interrelated constructs, both theoretically (Seligman, 2011) and empirically (Heshmati et al., 2020). We 
found that PERMA fits within the network model supporting hypothesis 1. Using exploratory graphical analysis allowed us to examine the extent to which elements in these two measurements cluster together and are hence deemed as overlapping and redundant. We found seven clusters within PERMA+NH and SWB. Not only did we find distinct clusters among PERMA $+\mathrm{NH}$, but we found no cross-loadings on SWB with the exception of positive and negative emotions on the PANAS questions. However, this is to be expected considering they are meant to measure the same construct. These results fail to support the idea that the elements of PERMA are the same as those in SWB or that PERMA elements are redundant, supporting hypotheses 2 and $3 a$.

The positive emotions and engagement clusters had small cross-loadings on meaning \& achievement supporting hypothesis $3 \mathrm{~b}$. These cross-loadings suggest that these components influence each other (see Christensen \& Golino, 2021; Christenen et al, 2020). You could think of it as a mediation model: the effect of the intervention on meaning $\&$ achievement is mediated by its effect on engagement. However, this implication assumes that PERMA+NH is a network model rather than a factor model. We find this assumption to be reasonable given the fit indices.

All together, conceptualizing the web of well-being provides two main contributions. First, it helps to understand the relationship between PERMA and SWB beyond latent correlations. This provides the perspective that PERMA is the decomposition of factors that work together to develop well-being. Second, it allows researchers a framework to examine the relationships between the components of PERMA+NH. We find a small effect between three of our clusters that may explain the high correlation among them. Viewing well-being as a network allows researchers and practitioners to conceptualize and examine elements of well-being as an 
interrelated "web" rather than blocks that build on top of each other. This approach better explains the theorized "connectivity" and "exclusivity" properties of PERMA (Seligman, 2011) through the examination of the degree of connectivity among items within and between elements of well-being.

\section{Strengths and Limitations}

In our examination of PERMA and SWB we acknowledge that our results are limited. First, while our sample was representative of the U.S. population, the web of well-being may not generalize to other, especially non-WEIRD (western, educated, industrialized, rich, democratic) countries. Theories of well-being have been criticized for being limited to WEIRD samples. Further research on PERMA and SWB on non-WEIRD samples is needed.

Network analysis has been considered an exploratory data-driven approach as opposed to hypothesis-driven. We conducted a confirmatory network analysis in addition to an exploratory analysis. However, in order to do this, we had to split our data in half. This reduced our sample for both analyses and may have reduced the accuracy of our results. Therefore, future examinations of the web of well-being should use confirmatory network analysis with the identified adjacency matrix (see osf.io/h2d4s/).

Critics of PERMA have noted that it is not exhaustive of the components of well-being. To overcome this we added two constructs found in the PERMA-profiler. However, even adding these may not complete our web of well-being. Adding additional constructs like those found in the PERMA+4 (environment, health, mindset, and economic security; Donaldson \& Donaldson, 2019) designed for the workplace may help to complete our framework. 
Another limitation is the results are based on cross-sectional data. While cross-sectional examination of well-being is informative, we are unable to model the relationship of PERMA and SWB over time. Furthermore, this also limits our examination of causal estimations of PERMA on SWB and PERMA components on each other. In fact, these network estimations may differ from person to person (Golino et al., 2022). Future work on SWB and PERMA should examine longitudinal data to better address these concerns.

\section{Conclusions}

While PERMA is not different from subjective well-being, we find that PERMA components are distinct from those found by measuring SWB alone. Viewing PERMA as a network creates a web of well-being. This proposed web of well-being helps to understand the interrelationships that take place to form well-being. 


\section{References}

Borsboom, D., \& Cramer, A. O. (2013). Network analysis: an integrative approach to the structure of psychopathology. Annual review of clinical psychology, 9, 91-121.

Christensen, A. P., \& Golino, H. (2020). On the equivalency of factor and network loadings. Behavior Research Methods, 1-18.

Christensen, A. P., Golino, H., \& Silvia, P. J. (2020). A psychometric network perspective on the validity and validation of personality trait questionnaires. European Journal of Personality, 34(6), 1095-1108.

Diener, E., Suh, E. M., Lucas, R. E., \& Smith, H. L. (1999). Subjective well-being: Three decades of progress. Psychological bulletin, 125(2), 276.

Diener, E., Wirtz, D., Tov, W., Kim-Prieto, C., Choi. D., Oishi, S., \& Biswas-Diener, R. (2009). New measures of well-being: Flourishing and positive and negative feelings. Social Indicators Research, 39, 247-266.

Donaldson, S. I., \& Donaldson, S. I. (2020). The positive functioning at work scale: Psychometric assessment, validation, and measurement invariance. Journal of Well-Being Assessment, 4(2), 181-215.

Donaldson, S. I., Heshmati, S., Lee, J. Y., \& Donaldson, S. I. (2020). Examining building blocks of well-being beyond PERMA and self-report bias. The Journal of Positive Psychology, $1-8$.

Epskamp, S., \& Fried, E. I. (2018). A tutorial on regularized partial correlation networks. Psychological methods, 23(4), 617. 
Fokkema, M., \& Greiff, S. (2017). How performing PCA and CFA on the same data equals trouble: Overfitting in the assessment of internal structure and some editorial thoughts on it.

Golino, H. F., \& Epskamp, S. (2017). Exploratory graph analysis: A new approach for estimating the number of dimensions in psychological research. PloS One, 12(6), e0174035.

Goodman, F. R., Disabato, D. J., Kashdan, T. B., \& Kauffman, S. B. (2018). Measuring well-being: A comparison of subjective well-being and PERMA. The Journal of Positive Psychology, 13(4), 321-332.

Golino, H., Shi, D., Christensen, A. P., Garrido, L. E., Nieto, M. D., Sadana, R., ... \& Martinez-Molina, A. (2020). Investigating the performance of exploratory graph analysis and traditional techniques to identify the number of latent factors: A simulation and tutorial. Psychological Methods, 25(3), 292.

Golino, H., Christensen, A. P., \& Nesselroade, J. R. (2022, August 2). Towards a psychology of individuals: the ergodicity information index and a bottom-up approach for finding generalizations. https://doi.org/10.31234/osf.io/th6rm

Hallquist, M. N., Wright, A. G., \& Molenaar, P. C. (2019). Problems with centrality measures in psychopathology symptom networks: Why network psychometrics cannot escape psychometric theory. Multivariate Behavioral Research, 1-25.

Kan, K.-J., Jonge, H. de, Maas, H. L. van der, Levine, S. Z., \& Epskamp, S. (2020). How to compare psychometric factor and network models. Journal of Intelligence, 8(4), 35.

Khaw, D., \& Kern, M. (2014). A cross-cultural comparison of the PERMA model of well-being. Undergraduate Journal of Psychology at Berkeley, University of California, 8(1), 10-23. 
McNally, R. J., Robinaugh, D. J., Wu, G. W., Wang, L., Deserno, M. K., \& Borsboom, D. (2015). Mental disorders as causal systems: A network approach to posttraumatic stress disorder. Clinical Psychological Science, 3(6), 836-849.

Pons, P., \& Latapy, M. (2005, October). Computing communities in large networks using random walks. In International symposium on computer and information sciences (pp. 284-293). Springer, Berlin, Heidelberg.

Schermelleh-Engel, K., Moosbrugger, H., \& Müller, H. (2003). Evaluating the fit of structural equation models: Tests of significance and descriptive goodness-of-fit measures. Methods of psychological research online, 8(2), 23-74.

Schmank, C. J., Goring, S. A., Kovacs, K., \& Conway, A. R. (2019). Psychometric network analysis of the Hungarian WAIS. Journal of Intelligence, 7(3), 21.

Slavin, S. J., Schindler, D., Chibnall, J. T., Fendell, G., \& Shoss, M. (2012). PERMA: A model for institutional leadership and culture change. Academic Medicine, 87(11), 1481.

Seligman, ME. (2011). Flourish: A visionary new understanding of happiness and well-being. Policy, 27(3), 60-1.

Seligman, Martin. (2018). PERMA and the building blocks of well-being. The Journal of Positive Psychology, 13(4), 333-335. https://doi.org/10.1080/17439760.2018.1437466

Seligman, M. E., \& Csikszentmihalyi, M. (2000). Positive psychology: An introduction. In Flow and the foundations of positive psychology (pp. 279-298). Springer. 
